NICHOLAS P. WHITE

\title{
WHAT NUMBERS ARE
}

"We will take as our starting-point the series $0,1,2,3, \ldots, n, n+1, \ldots$, and it is this series that we shall mean when we speak of the 'series of natural numbers'." This is Russell's announcement, on the third page of his Introduction to Mathematical Philosophy, of his discussion of natural numbers. But, he asks, - like many before and since - what are these things, the numbers? He gives the 'logicist' reply that they are sets, and many have agreed. For they have seen that, as is well known, arithmetic can be modeled in set theory. But not only can arithmetic be modeled in set theory. It can be thus modeled in infinitely many different ways. Thus different ways of modeling arithmetic appear to make the number three, for example, turn out to be quite different sets. This copious supply of models might seem an explicative embarrassment of riches, and some so take it. Thus, Quine maintains, "any progression will serve as a version of number", with the obvious qualification: "so long and only so long as we stick to one and the same progression" (Quine, 1969, p. 45). So each natural number may be taken to be a set, though of course a given number may not be taken simultaneously to be different sets. ${ }^{1}$

It has been maintained, however, that this very profusion of settheoretic models of arithmetic makes it impossible to say that numbers are sets. Thus Benacerraf argues that, in brief, since there are infinitely many set-theoretic accounts of what numbers are, all of which capture the notions of arithmetic equally well, there is therefore no good reason to say that numbers are sets at all. For, after all, "if numbers are sets, then they must be particular sets, for each set is some particular set" (Benacerraf, 1965, p. 62).

In the face of these two roughly opposing views, I should like to try a different tack - at least I think that it is a different tack, and that what I shall end up saying is distinct from the views of both Quine and Benacerraf. 
Before I begin, however, let me point out some of the limits which are here imposed, partly by my own prejudices, upon my project. The desire to claim that numbers are sets has often historically been tied up with the doctrine that mathematics is deducible from logic, and this doctrine in turn has been tied up with the doctrine that logic, including set theory, is in some way more basic, epistemologically or metaphysically, than mathematics. Both of these doctrines, moreover, have links with the view that mathematics is necessary, or knowable a priori, or analytic, or all three, and with the desire to show that this is so. I want to make it clear at the outset that such doctrines and views are not my concern here. The reason is partly that most of the notions which figure in them are not, to my mind, clear or easy to operate with. Thus, for example, I am not comfortable with the question whether arithmetic is more or less 'basic' than set theory. In the second place, however, - and for reasons which I cannot hope to expound here - I do not believe that the question whether numbers are, say, sets is dependent upon the doctrines and views just raised. In particular, I do not believe that the question whether natural numbers are sets is affected by the issue of the relative 'basicness' of arithmetic and set theory. If the issue which I shall discuss seems accordingly denuced here of its usual philosophical covering, I can only apologize for its immodesty.

II

If the existence of a model of arithmetic in set theory was ever a reason for identifying numbers with sets, then, I maintain, the existence of multiple set-theoretic models of arithmetic should prompt us, not to say with Benacerraf that numbers cannot be sets, but rather to suggest that there are multiple full-blown series of natural numbers. Thus, for example, instead of there being only one three, there are after all many threes, and many thirty-sevens, and so on. This suggestion constitutes the central theme of this essay.

The first step of the suggestion is to suppose that we can, in our arithmetical discourse, replace our numerical singular terms, such as 'three', by corresponding atomic general terms, such as 'is a three', or 'threes'. Now it might be objected immediately that the suggestion can make no sense, on the ground that 'three' and its like are singular terms in English. But this objection seems weak. Even if 'three' is taken to be a singular 
term in English, it seems to me plain that the suggestion does make sense - just as much sense as the claim, which might be made by someone who had concluded that the so-called Homeric poems were produced by different people, that there turn out to have been several Homers. Further support for the intelligibility of such claims (though not an explanation of it, which I do not propose to try to provide) is forthcoming if we consider Quine's way of eliminating singular terms other than variables (Quine, 1960 , pp. $179 \mathrm{ff}$.). If his ' $(7 x)$ ( $x$ Socratizes)' is an acceptable explication of 'Socrates', then equally ' $(~(x)$ ( $x$ threes)' would be an acceptable explication of the singular term 'three'. But if this is so, then it makes sense to ask how many things satisfy the predicates ' $x$ threes', and to answer that, after all, many do.

Postponing for now further issues and objections, let us consider further, briefly and informally, what the suggestion itself amounts to. I have proposed replacing the putative singular terms ' 0 ', ' 1 ', ' 2 ', ' 3 ',,. , by a set of general terms. The intuitive idea behind the suggestion is that to be a three, e.g., is to stand in a certain position (viz., fourth, counting zero) in some progression or other. Plainly, what we therefore want is a relativized notion of what it is to be a three - relativized, that is, to progressions. (This and subsequent relativizations capture the idea which Benacerraf expresses when he says that objects do not do the job of numbers 'singly'; see his p. 69). Thus what we want to catch are the notions expressed by the binary predicates ' $x$ is a zero in $p$ ', ' $x$ is a one in $p$ ', and so forth, where ' $p$ ' ranges over progressions, all the while recognizing that a given object might stand in a certain position in one progression in spite of occupying other positions in other progressions.

How are we to explain such binary predicates as ' $x$ is a three in $p$ '? The idea behind this predicate, we know, is that $x$ stands fourth in $p$. Ordinarily, this idea might be explained by saying that the precursors of $x$ in $p$ can be mapped one-to-one onto the numbers (again including zero) through two. But remember that we are now attempting to avoid using number words as singular terms, and thus we can not remain happy with this explanation. Fortunately, however, we can avoid the difficulty in a manner originated by Frege. For example, we may explain ' $x$ is a zero in $p$ ' by saying that there is no precursor of $x$ in $p$. (Note that in putting the point thus I am using a compressed manner of speech: what we have to say strictly is that there are no elements of $p$ which precede $x$ 
in $p$, since there may of course be elements of $p$ which, while they come before $x$ in other progressions, do not do so in $p$.) Then ' $x$ is a one in $p$ ' can be explained by saying that there is exactly one precursor of $x$ in $p$, which can in turn be explained in familiar fashion by saying that there is a precursor, $y$, of $x$ in $p$ and there is no precursor of $x$ in $p$ which is not identical with $y$. And so forth for all of the binary predicates we need. We can then, of course, go on to construct such general terms as ' $(\exists p)(x$ is a three in $p)^{\text {'. }}$.

Let me pause briefly in the account to make a few points. First, my suggestion is to be sharply contrasted with Benacerraf's claim, which is that numbers are not objects. My view, on the contrary, is that numbers are objects, namely objects which occupy positions in progressions, and of which such predicates as ' $\exists p)(x$ is a three in $p)$ ' are true. (Ironically, however, we shall see that my suggestion might be modified, in a Benacerrafian spirit, into a denial that numbers are objects.) Second, my suggestion is to be distinguished from another quite different view, that the number three, e.g., is the set of all objects which stand fourth in some progression or other. This view would not be a happy one, since if absolutely any object whatever can be placed fourth in some progression or other, then the suggested set would be the universal set, and paradox would ensue. (But my suggestion itself will still, later on, have to avoid the threat of set-theoretic paradox.) Moreover, aside from worries about paradox, such an identification of numbers with sets would be just one set-theoretic explanation of number on a par with all of the others, and there would be nothing in arithmetic to dictate a choice of it over the alternatives. ${ }^{2}$ Third, notice that my suggestion seems also to carry a certain threat of Pythagoreanism. For if we did say that any object could stand in a progression, then my suggestion would seem to imply that every object is a number. I shall pursue this point later, where we shall find that we can turn it to advantage.

Given our binary numerical predicates, it is easy to see how the rest of the details will work. Statements of pure number theory such as $' 3+2=5$ ' will emerge as general statements about progressions. Thus in this example we end up saying that give any progression, $p$, the sum in $p$ of a three in $p$ and a two in $p$ is a five in $p$. Note that I say "the sum in $p$, since depending on the progression in which two things are taken, their sum can turn out to be quite different objects, standing in quite 
different positions, and moreover one or both of them will be absent from certain progressions.

This fact in turn reminds us that such arithmetical notions as that of 'sum' must also be relativized to progressions, so that we can say, e.g., ' $z=$ the sum of $x$ and $y$ in $p$, or for short, ' $z=\operatorname{Sum}(x, y, p)$ '. The traditional joint conditions on the notion of 'sum' are:

(1) The sum of $n$ and 0 is 0 ,

and

(2) The sum of $n$ and the successor of $m$ is the successor of the sum of $n$ and $m$.

Our analogue of (2) is:

$\left(2^{\prime}\right) \quad(x)(y)(p)$ (if $x$ is in $p$ and $y$ is in $p$, then $\operatorname{Sum}(x$, the successor of $y$ in $p, p)$ is identical with the successor in $p$ of $\operatorname{Sum}(x, y, p)$,

while corresponding to (1) is:

$\left(1^{\prime}\right) \quad(x)(y)(p)$ (if $x$ is a zero in $p$ and $y$ is in $p$, then $\operatorname{Sum}(x, y, p)$ is identical with $y$ ),

in the latter of which we must rely on the explanation just given of ' $x$ is a zero in $p$. Other arithmetical notions are similarly tractable.

Statements of 'applied' number theory can also be straightforwardly handled. First of all, there are statements of the form 'There are $n F \mathrm{~s}$ ', which will be considered later, where it will be important that they do not oblige us to treat numbers as objects - i.e. to quantify over them - at all. (Just notice here, however, that our versions of such terms as 'the number of $F \mathrm{~s}$ ', when we have occasion to use them, will be different from the ordinary, since for us there is no such thing as the number of $F \mathrm{~s}$, but only 'the number in $p$ of $F \mathrm{~s}$ ', that is the object, $x$, in $p$ such that there is a correlation of $F$ s with the precursors in $p$ of $x$; but more of this shortly.) Secondly, however, there are statements which do require the use of quantifiable variables ranging over numbers. This requirement can be escaped in the case of 'There are as many $F \mathrm{~s}$ as $G \mathrm{~s}$ ' through the rendering of it by 'There is a correlation of the $F \mathrm{~s}$ and the Gs' (though this escape leads to no great ontological saving, since we are then required 
anyway to quantify over relations; cf. Quine, 1960, p. 269). But if we want to say that there are twice as many $F \mathrm{~s}$ as $G$ s, we should ordinarily have to say, e.g., that for some number $n$ (not zero) there is a correlation of the $G$ s with the numbers less than $n$, and a correlation of the $F \mathrm{~s}$ with the numbers less than the sum of $n$ and $n$. Our scheme, on the other hand, would have us say correspondingly that every progression $p$ contains some element $x$ (not a zero in $p$ ) such that there is a correlation of the $G \mathrm{~s}$ with the precursors in $p$ of $x$, and a correlation of the $F \mathrm{~s}$ with the precursors in $p$ of the sum in $p$ of $x$ and $x$. Other such statements can be treated analogously.

The discussion of the previous section has shown informally how to explain our new numerical terms and how to render arithmetical statements in our new scheme. What the discussion shows is that there is nothing in the discourse of arithmetic itself which requires us to fix on a single object, whether set or otherwise, as the number three, e.g., or on any single progression as the progression of natural numbers. As far as that discourse is concerned, we may have more threes, and more sequences of numbers, than we had thought we had. ${ }^{3}$

I propose now to take up some objections which might be raised against the view which $I$ have suggested, and to indicate the lines of response to them which I would take. In certain cases the indication will be sketchy, because some of the objections introduce issues far too broad to be treated fully here.

Consider first Benacerraf's conclusion, that numbers are not objects. Why should my suggestion be preferred to his? In the first place, his view appears to have an unnatural feature, namely that he denies that numbers are objects while seemingly continuing to use number-words and numerals as singular terms. Secondly, he does not tell us very much about what his numerical non-objects might be like. In fact, however, I cannot rule out $a$ priori the chance that these defects might be remediable, and one who is partial to his view can well view my suggestion as pressure to produce the remedy. Indeed, my suggestion itself might be used as an ingredient. For one who accepts the construal of number-words as general terms, and who also adopts the Fregean view that general terms denote 'concepts' (as distinct from 'objects'), might conclude that numbers are 
concepts in Frege's sense. I might myself adopt this view, but for the fact that there seem to me to be difficulties in the Fregean notion of a concept.

The second line of objection will take a little longer. It is easy to suppose that although many different progressions satisfy the laws of arithmetic, there is nevertheless one progression, considered to be the sequence of natural numbers, which is special in that it somehow primarily satisfies those laws, and that it is somehow merely mimicked in so doing. This supposition seems to me plainly false. As far as merely satisfying arithmetical laws is concerned, all progressions are on a par. More generally, the point can be put by saying that unintended models of a formal system, however unintended they may be, satisfy the system just as much as intended models do.

But even if this much is accepted, the objection may persist that a certain single progression is still special, in that it is to members of it that we in fact refer when we use numerals or number-words. I find this claim dubious too, for reasons which will be familiar and congenial to those who are sympathetic with Quine's views on ontological relativity (see Quine, 1969, p. 54), since it is unclear to me how we can so confidently identify the referents of those arithmetical words. Moreover even if we could identify them, it would not therefore be evident that a certain progression would be shown to be special in a way which would counter my suggestion, or show that there are not, after all and in spite of how we have referred heretofore, numerous sequences of numbers. For even if we take the references of numerical expressions in our ordinary arithmetical discourse to be fixed, the presence of multiple models of arithmetic makes us realize that we could equally well have referred differently and still have come out with arithmetical truths.

Accept even this much, though, and a new version of the objection appears, to the effect that regardless of how we might refer, there is still a single, special, progression to which we somehow should refer in making our arithmetical claims. Though it may be my fault, I have difficulty here in following the objection, since I do not see what might support this contention. Thus, for example, I do not see how anything about arithmetical discourse is explained by singling out a single progression as its proper subject-matter. ('What about counting?' someone will ask; but counting will be taken up shortly.) 
But the line of objection which I have in mind still has other courses which it may take. For instance it might be said that my suggestion errs in supposing that the question what numbers are can be answered by taking arithmetic as an uninterpreted formal system and asking what structures satisfy it. Rather, it could be said, one can answer the question only by taking arithmetic as understood, and by somehow reproducing its content in terms with the same meaning. Or, in a perhaps similar vein, it can be asserted that the approach which I have adopted falls short of saying what numbers 'really' are. My view is that this line of thought is mistaken, but I obviously cannot settle the point here, since the issue is now a broad and deep one regarding the notion of explication in general, and regarding the whole project of 'saying what so-and-sos are'. Two brief points, however. First, I do not believe that this project need or should involve requirements of sameness of meaning. Second, this line of argument is not directed exclusively against my suggestion, but would be equally cogent, if it is cogent at all, against many accounts of number which appeal to only a single progression.

I might also mention - though again I broach an issue which I shall not follow up - that I do not construe my suggestion as 'reducing' numbers to other objects in the sense of denying that there ('really') are numbers. On my view, it seems to me, there are indeed numbers, and plenty of them.

Let me now move to a third sort of objection. Our story obliges us to quantify over progressions and the objects in them. But what are we taking as the range of the variable ' $p$ '? Plainly we must take care, since if we say that it is to range over absolutely any and all progressions of absolutely any and all objects, then we shall be headed for set-theoretic paradox. We must therefore choose some restriction on the range of ' $p$ ' (along with suitable restriction on the range of ' $x$ ', etc.). It is well known, however, that no unique choice is forced upon us, and that different choices would equally avoid paradox. Thus, we have various choices - short of the naive and unrestricted but self-contradictory one - of what progressions and elements to say that there are. But, under our account, these choices bring with them choices of what so say numbers are. For even if two people accept our account so far, they will still disagree over what numbers are if they differ in their background views concerning what progressions there are. 
The objection therefore arises to our account that it places us back exactly where we started, when we were worrying over the multiple models of arithmetic. ${ }^{4}$ For it will be urged that, just as before, we are without a flat identification of numbers with a fixed group of objects, but instead have an array of alternatives from which we must select. The heir to Benacerraf's argument will then urge us to accept the thesis that numbers are not objects.

Although this objection shows that there is a sense in which we cannot claim to have said flatly what numbers are, it does not demonstrate that we are back where we began. We cannot say flatly what numbers are because we cannot specify a unique correct range for ' $p$ '. What we have, rather, is in the nature of a schema for the explanation of what numbers are: that is, we have a way be which anyone, having constructed his consistent theory with all of the progressions which it allows, may explain in his own theory what things the numbers are. At the start, by contrast, our problem was that even given a consistent background theory with some progressions, we still could not make a non-arbitrary choice from among alternative accounts of numbers. We no longer have that problem. Our problem now is instead the broader one - which we had on our hands anyway - of deciding how to avoid the paradoxes of naive set theory. But relative to a decision on this matter (however arbitrary the decision may be), we can now identify the numbers without any additional arbitrariness.

A related objection is to the effect that, since our account can be viewed as a reduction of arithmetic to that part of set theory dealing with progressions, it therefore stands on a par with all of the other settheoretic accounts of number - notwithstanding the fact that it avoids identifying numbers with particular sets. Moreover the objection can be bolstered by citing the ontological price which our account exacts, since unlike other accounts, ours will standardly involve quantifying over progressions. Here again we touch upon broad and deep issues, not to be explored here. Suffice it to say that this ontological profligacy can be balanced off against a virtue of the account. This virtue lies in its having - it seems to me - a certain elegance, and in its ability to help us avoid the arbitrariness, just alluded to, of plumping for one possible identification of numbers without any very strong reason for doing so. Likewise it provides a perspicuous rationale for switches which we may occasionally 
make, for the sake of technical convenience, from one set-theoretic account of number to another, since it enables us to view ourselves, not as changing our assumptions about what numbers are, but simply as working with a fresh stock of numbers.

IV

Counting. Some will argue that it has been wrongly ignored so far, and that once it has been adequately treated, there will be no doubt that in counting we must use numerical singular terms, and that there is only one sequence of natural numbers. This objection can be briefly countered. As is well known, statements involving finite cardinalities can be made without treating numbers as objects at all, simply by using quantification and identity to define numerically definite quantifiers in the manner of Frege (see Quine, 1960, p. 268, and recall our use of the same device to define our binary numerical predicates). But if we do not need objects here at all, we do not need a unique sequence of them either. ${ }^{5}$

Though the argument might end there, there is still some interest in the following fact, that even if in one's counting and cardinality-assignments one uses numerical singular terms, there is nevertheless no need to suppose that there is only a single sequence of numbers. Let us briefly digress on the matter.

On a widespread view, counting amounts to a kind of mapping, namely certain mappings of sets onto initial segments of the sequence of numbers. Suppose that this is so, and it still does not follow that there is only one sequence of numbers. We may adapt several points made above (in Section III, a propos of the 'second objection'). First, one may properly have doubts about how we may identify the referents of the terms used in counting, and be sure that they belong to a certain single progression. Second, even if we suppose that we in fact use a certain progression, we can still recognize that we could have used a different one. And not only could that different progression have been used for counting various and sundry sets; it could also have been used for counting segments of the progression which we suppose ourselves actually to be using. I see no reason to say that such an operation would not be genuine counting, or that anything about counting is explained by the idea that it must be performed on a particular instrument. 
There is a similar contention to be made about a view of Benacerraf (pp. 71-72), that "in counting, we do not correlate sets with initial segments of the numbers as extra-linguistic entities, but correlate sets with initial segments of the sequence of number words", and that for purposes of counting we may perfectly well do without the supposition that 'two', '2', 'zwei', and 'deux', all stand for the same object. But while he conceives of counting as mapping of sets onto numerical expressions, he apparently also thinks that we ought to adopt or view a single such string as somehow basic. I doubt that we do so or need to do so. Many of us are quite capable of operating with the linguistic sequence ' $0,1,2, \ldots$ ' or with the linguistic sequence 'zero, one, two,...', and perhaps with others as well. I see no good reason to think that one of these strings of expressions is more basic, or that one of them must be more basic in order for us to understand both of them. All that seems to be required is that we all be able uniformly and with facility to map each sequence onto the other in an order-preserving way. Thus, not only could we use different linguistic progressions from any one which we in fact use; we actually do use different such progressions on different occasions.

The point still holds mutatis mutandis if we switch back to viewing counting as a correlating of sets with non-linguistic entities referred to by numerical expressions. For what is impossible about the idea that a man could count in this way using either of two progressions indifferently, and what would show that one of them must be basic, to his mind or otherwise?

$\mathrm{V}$

I remarked earlier that our account might appear to make out that everything is a number. Let us see in what sense this might be so.

This Pythagoreanistic look first comes over our account when we consider Benacerraf's remark that any object may (as he puts it) 'play the role of', say, the number three, since any object whatever may occupy the fourth - or indeed, any - position in some progression or other. Thus, we seem to have a staightforward sense in which each object is a number: each object is an element in some structure satisfying arithmetic. (If we insist on dealing with counting, we could still say '.. and is usable for counting' without running afoul of Benacerraf's claim that only certain progressions can be counted with; cf. Note 5.) 
But the matter must not be taken to be more straightforward than it is. It is not as though we had some single theory in which each object is an element in some progression, and we have observed that the hope of getting one must be eschewed if we are to avoid paradox. It is rather that even if an object is not, in a particular theory, ranged in a progression, still it could be so ranged in an adjusted theory, provided of course - more on this proviso shortly - that we can speak of the same object in more than one theory.

Keeping this Pythagorean shadow in view, consider now Quine's treatment (in Quine, 1966 and 1969) of the Löwenheim-Skolem theorem, which tells us that any theory with a true interpretation has a model in the natural numbers (I am dealing here with the sort of theories which Quine has in view). What worries Quine about the theorem is Berry's suggestion that it leads us to Pythagoreanism, that is, to the conclusion that natural numbers are all that there is. My concern with this problem here is not with what is often called 'Skolem's paradox', the supposed oddity in the fact that non-denumerable models of denumerable theories can, as it were, be pared down to denumerable models. Rather, it is with the idea that the denumerable models of theories can be construed as models in the natural numbers (Quine, 1969, p. 59), since it is here that Pythagoreanism enters in. I suggest that what we have seen so far gives us an approach to the Löwenheim-Skolem theorem, and makes its attendant Pythagoreanism seem less odd than it might. If this is so, then so much the better for our account of number.

Skolem's 'paradox' aside, what seems so odd about the theorem is that it seems to go against our conviction that there are many objects which just are not numbers. Now, however, we have a way of looking at any object under which it is not unnatural to view it as a number, since we know that we could (subject to the proviso noted two paragraphs back) place it in a progression which models arithmetic. But we must be careful here. The theorem tells us that each object to which a theory can be taken to refer may be construed to be a natural number in a structure which, as a whole, satisfies that very theory. As we have seen, however, our account of number does not say that each object to which a theory can be taken to refer must necessarily, within that very theory, be ranged in some progression. Nor - to repeat - does it say that there is a single theory within which all objects whatever stand in progressions. Never- 
theless, it does seem to me that some of the oddity about the theorem is alleviated once we see how each object of a theory, so to speak, may be seen, albeit in some other theory, as a number, that is, as an element in a progression.

This way of approaching the Löwenheim-Skolem theorem rests, as we saw, on the supposition that the same object can be spoken of in more than one theory. Now Quine's discussion of ontological relativity and of the inscrutability of reference rightly questions this supposition. Does this fact cripple this approach to the philosophical problem raised by that theorem? No, because the problem itself cannot be posed without the same supposition. In Quine's terminology, to construe a theory as being about numbers we must presuppose both a background theory and a manual for translating into that background theory the theory which we are examining. (Indeed, this fact is recognized in Quine's own way of disarming the problem by means of his own doctrine of ontological relativity, under which "there is no absolute sense in speaking of the ontology of a theory"; see his 'Ontological Relativity', p. 60.) But likewise that is all that is being presupposed when we identify an object, which is not in one theory ranged in any progression, with an object which is so ranged in another theory. And it would seem arbitrary here to countenance enough intertheoretic identification of objects to generate the problem, but not enough of the same sort of intertheoretic identification to alleviate it. ${ }^{6}$

\section{The University of Michigan}

\section{NOTES}

1 Note in this connection that the claim that any progression satisfies arithmetic is different from the claim that any structure satisfying arithmetic is a progression. The falsity of this latter claim will not affect my argument.

2 Here I am indebted to Kim.

${ }^{3}$ It seems to me likely that analogous moves can be made in connection with similar philosophical problems.

I should point out that I have said nothing about how naturally or unnaturally the above account might fit in with more general accounts of broader classes of numbers. On this score, however, I see nothing very threatening.

4 Here I am indebted to Dreben.

5 Let me by the way make explicit a point which has, I think, been somewhat hidden in discussion of number and counting. It is that there are two distinguishable though related questions a foot: (1) whether a correct account of number requires an account 
of counting (see Quine, 1960 , pp. 262-263, vs. Benacerraf, 1965, p. 51, Note 3, and p. 72), and (2) whether a correct account of counting requires talk of numbers as objects (Quine, ibid., p. 268). Quine answers both questions in the negative; Benacerraf's answer to the first is affirmative, and he makes no explicit response to the second (I myself agree with Quine on both). Although the two questions are linked, it is important to avoid conflating them. For example it appears to me that such conflation is partially responsible for the mistake in Russell's argument for an affirmative answer to the first question (Russell, 1919, p. 10).

Benacerraf also believes that any progression usable for counting must have a 'less than' relation which is recursive, and therefore he believes that some progressions cannot do the job of numbers (pp. 51-53). Since I think that we can ignore counting, I shall leave this issue aside. However, Benacerraf's restriction on admissable progressions could easily be accommodated in what follows.

${ }_{6}^{6}$ I owe thanks to many, including W. D. Hart, for discussion on the topic of this paper, and also to many for their helpful comments on earlier drafts, including Paul Benacerraf, Burton Dreben, W. V. Quine, and especially Jaegwon Kim.

\section{REFERENCES}

Benacerraf, P.: 'What Numbers Could Not Be', Philosophical Review 74 (1965), 47-73. Quine, W. V.: Word and Object, The MIT Press, Cambridge, Mass., 1960.

Quine, W. V.: 'Ontological Reduction and the World of Numbers', The Ways of Paradox and Other Essays, Random House, New York, 1966, pp. 199-207.

Quine, W. V.: 'Ontological Relativity', Ontological Relativity and Other Essays, Columbia University Press, New York, 1969, pp. 21-56.

Russell, B.: Introduction to Mathematical Philosophy, G. Allen, London, 1919. 